\title{
Coherence manipulation of partially mode-locked fiber laser
}

\author{
Tao Zhu ${ }^{1, *}$, Lei Gao ${ }^{1}$, Stefan Wabnitz ${ }^{2}$, Min Liu ${ }^{1}$, and Wei Huang ${ }^{1}$ \\ ${ }^{1}$ Key Laboratory of Optoelectronic Technology \& Systems (Ministry of Education), Chongqing University, Chongqing 400044, China \\ ${ }^{2}$ Dipartimento di Ingegneria dell'Informazione, Università degli Studi di Brescia and INO-CNR, via Branze 38, 25123 Brescia, Italy. \\ E-mail address: zhutao@cqu.edu.cn
}

\begin{abstract}
We find that adjusting the polarization state controls the process of loss of coherence of partially coherent laser, where parametric frequency conversion populates longitudinal modes with a random distribution of position, intensity and polarization.

OCIS codes: (140.3510) Lasers, fiber; (030.1640) Coherence; (260.5430) Polarization
\end{abstract}

\section{Introduction}

Transition from highly coherent to weakly coherent states is common in most natural nonlinear systems, such as in optics, Bose-Einstein condensation, semiconductors, and fluids. Stochastically driven nonlinear processes limit the number of amplified modes in a natural system due to competitive mode interaction, which is accompanied by loss of coherence when increasing the complexity of system [1-3]. Here, we demonstrate a new pattern formation process in partially mode-locked fiber laser (PML), where the laser redistributes its energy from center wavelength to sidebands through parametric instability (PI), and subsequently longitudinal modes are populated via cascaded four-wave-mixing (FWM). We find that the PI, which exhibits great fluctuations when it is spontaneously grown from noise in conservative systems [2], may possess a high degree of coherence in dissipative laser system with gain, and quasi-periodic cooperative and competitive cascade FWM-activated mode interactions are obtained. The polarization of each filtered wavelength of PML tends to dithering/vibrating, indicating that the well-defined state of polarization (SOP) of conventional dissipative solitons is also broken in PML. This new route is beneficial in understanding of supercontinuum, Kerr-combs phenomena, and optical rogue waves.

\section{Experimental results}

The fiber ring cavity consists of $1 \mathrm{~m}$ erbium-doped fiber, $19.5 \mathrm{~m}$ dispersion compensation fiber, $14.5 \mathrm{~m}$ single mode fiber with dispersions of $15.7,-38$, and $18 \mathrm{ps} / \mathrm{nm} / \mathrm{km}$, respectively (more details are in Ref. 4). We inset a saturable absorber, which is fabricated by filling reduced graphene oxide flakes into cladding holes of a photonic crystal fiber, to detuning nonlinear phase for phase matching through adjusting the SOP of light. The output is recorded by conventional devices, together with Disperse Fourier transformation for single-shot detection.
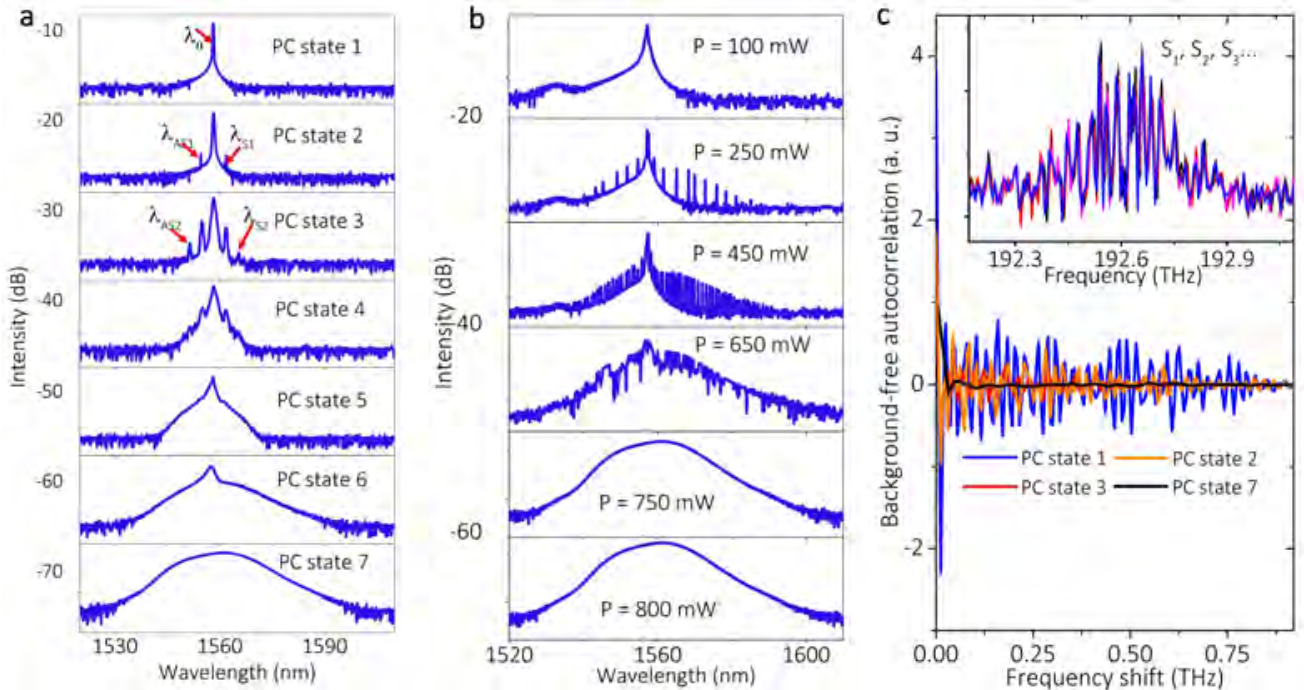

Figure 1. (a) Averaged optical spectra evolution of PML for different PC states. (b) Averaged optical spectra for different pump power levels, where the PC is fixed at state 7. (c) Autocorrelation analysis of the first Stokes region in single-shot spectra for different PC states. The inset contains 5 consecutive single-shot spectra within the first Stokes region under PC state 2, where only small fluctuations are shown.

Rotating polarization controller (PC) carefully, we observe stable PML regime with easiness. Figure 1 (a) depicts averaged optical spectra under different PC states, suggesting that the continuous wave $(\mathrm{CW})$ laser is destabilized by 
PI at first, then additional longitudinal modes are activated through cascaded FWM among different PI gain lobes, finally leading to PML when further optimizing the intracavity polarization evolution. The averaged optical spectra suggest that at first the CW laser is destabilized by PI. Next, additional longitudinal modes are activated through cascaded FWM among different PI gain lobes, finally leading to PML by further optimizing the intracavity polarization evolution. The center mode and the four new sideband mode wavelengths, namely, $\lambda_{2 \mathrm{AS}}, \lambda_{1 \mathrm{AS}}, \lambda_{1 \mathrm{~S}}, \lambda_{2 \mathrm{~S}}$, all satisfy the energy conservation of FWM. The PI sideband spacing is $0.438 \mathrm{THz}$, which is in relatively good agreement with the calculated value for the estimated intracavity peak power level of $\sim 5 \mathrm{~W}$ of the central mode quasi-CW pulses.

We investigated the coherence of the PI gain lobes based on the statistical analysis of spectral correlations [2] The background-free correlations of the first Stokes region $(0.9 \mathrm{THz})$ for different PC states are shown in Fig. 1 (c). As can be seen, our results exhibit a great difference with respect to the case of MI in a conservative system. Quasiperiodic fluctuations of the autocorrelation centered at zero indicate cooperative (positive value) and competitive (negative value) interactions of the individual cavity modes. Most importantly, both the positive center peak with zero frequency shift and the deep negative valley diminish gradually with polarization detuning. The same properties are observed in other Stokes and anti-Stokes regions. Yet, this correlation disappears when considering the whole spectrum. When PML is formed, the autocorrelation trace of the first Stokes region finally evolves into to a flat zero-background, indicating that each mode is only correlated with itself. Such loss of correlation provides the representation of the loss of coherence.

Tuning of pump strength may also form PML. Keeping the PC in state 7, we record the averaged spectra in Fig. 1 (b), and find that more longitudinal modes are activated when pump strength grows larger: correspondingly, irregular pulses with high intensities are formed. Tuning of the pump strength may also form PML. More longitudinal modes are activated when the pump strength grows larger: correspondingly, irregular pulses with high intensities are formed. The observed temporal asymmetry grows larger for stronger pumping levels. For low pump strengths, the pulse packets are localized between zero intensity regions (i.e., they are located in correspondence with the giant quasi-CW pulse). On the other hand, more packets appear when the pump power is high enough. For example, whenever the pump power is set at $750 \mathrm{~mW}$, pulse packets merge with each other. Even higher pump powers flatten the pulse energy in each round-trip by coupling among different frequencies. Those properties clearly illustrate the onset of intracavity parametric frequency conversion, a nonlinear process that exhibits optical bistability when the phase matching is optimized. Phase-locking of longitudinal modes leads to a hysteresis loop for the average output power as the pump power is varied in opposite directions. The periodicity of the pulse packets leads to the appearance of discrete frequency components in the average optical spectra, due to the limited detection time of our OSA.
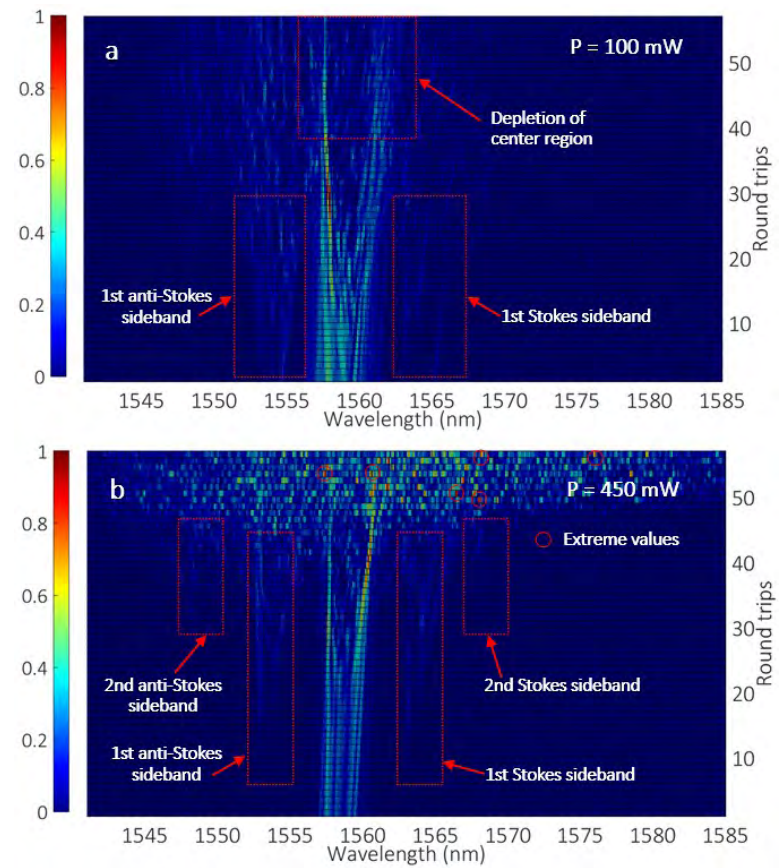

Figure 2. Single-shot spectra for pump powers of (a) $100 \mathrm{~mW}$ or (b) $450 \mathrm{~mW}$, respectively, where the PC is fixed at state 7. 
The single-shot spectra in Fig. 2 reveals that the CW mode is depleted when the pump power is relatively small, which deteriorates the phase matching condition of PI. However, the $\mathrm{CW}$ transfers its energy quickly to sidebands for larger pump powers. This is accompanied by a sudden evolution from a narrow CW spectrum to a set of broad but randomly distributed comb lines. As can be seen in Fig. 2 (a), quasi-CW and narrowband spectra are generated over the first few round trips, until new sidebands appear on both the Stokes and the anti-Stokes side after further laser circulations. The initial spectral broadening around the initial $\mathrm{CW}$ mode originates from self-phase modulation (SPM): here the spectrum remains relatively smooth until the generation of sidebands occurs. For round trip numbers larger than 40, the depletion of the quasi-CW region is clearly observed, in correspondence with the generation of the distant sideband frequencies. Here, only 1st order Stokes and anti-Stokes sidebands are generated, and the depletion of the central quasi-CW pump mode region makes it hard to generate the 2nd order Stokes and anti-Stokes sidebands. Pump mode depletion deteriorates the QPM phase matching condition of PI, and leads to an abrupt switching to CW lasing.

The second characteristic is that, for relatively large pump powers, the $\mathrm{CW}$ mode quickly transfers its energy to high-order sidebands. This process is accompanied by a sudden evolution from a narrow quasi-CW spectrum into a set of broad but randomly distributed comb lines, as shown in Fig. 2 (b). The multi-wave mixing process among the sidebands and central pumping region produces a randomized spectrum at each round trip. As the number of round trips necessary for accumulating a significant nonlinear phase shift decreases with increasing pump strength, the larger the pump power, the quicker the generation of pulse clusters. When the pump power is large enough, a broad comb with randomly spaced lines can be generated even within a single round trip.

The loss of coherence is also reflected in the SOP of the filtered PML output. Primarily, we observed that the SOP of the center wavelength remains a fixed point on the Poincaré sphere, while wavelengths far away from the center mode bifurcate into a cross-like configuration of output SOPs. Furthermore, irregular polarization states located outside of the main polarization directions emerge for red-shifted sidebands whenever the pump power reaches 250 $\mathrm{mW}$ : SOP scattering aggravates over the whole wavelength span for the pump power of $450 \mathrm{~mW}$. We attribute the polarization deviation from the cross shape to chaotic competition of cascaded FWM processes. When the pump power is larger than $600 \mathrm{~mW}$, additional longitudinal modes are populated, and the SOPs of filtered wavelengths appear as totally random, owing to enhanced spectral energy transfer processes. This randomization of output SOP is associated with the loss of temporal coherence.

\section{References}

[1] J. M. Dudley, F. Dias, M. Erkintalo, and G. Genty, "Instabilities, breathers and rogue waves in optics," Nat. Photon., 8, 755-764 (2014).

[2] D. R. Solli, G. Herink, B. Jalali, and C. Ropers, "Fluctuations and correlations in modulation instability," Nat. Photon., 6, 463-468 (2012).

[3] A. Runge, C. Aguergaray, N. Broderick, and M. Erkintalo, "Coherence and shot-to-shot spectral fluctuations in noise-like ultrafast fiber lasers," Opt. Lett., 38, 4327-4330 (2013).

[4] L. Gao, T. Zhu, S. Wabnitz, M. Liu, and W. Huang, “Coherence loss of partially mode-locked fiber laser,” Sci. Rep., 6, 24995 (2016). 